\title{
Perilymphatic Fistula Following a Closed Head Injury
}

\section{Zolnourian $\mathrm{A}^{*}$ and Paramanathan V}

Department of ENT, Queen's Hospital Burton, Belvedere Rd, Burton-on-Trent, Staffordshire, UK

\begin{abstract}
We present a case of sudden sensorineural hearing loss and paroxysmal positional vertigo following a closed head injury. A perilymphatic fistula (PLF) was suspected in the absence of any other definite diagnostic findings The patient underwent exploratory tympanotomy. Fistula was grafted which noticeably improved his vestibular symptoms. PLF must be considered in post-traumatic sensorineural hearing loss with or without other vestibular symptoms. Surgical treatment with patching of oval and round windows remains an effective treatment, but its effect on hearing loss is less predictable.
\end{abstract}

Keywords: Perilymphatic fistula; Closed head injury; Sensorineural hearing loss; Tympanotomy

\section{Introduction}

PLF is a condition where there is an abnormal communication between the fluid-filled perilymphatic space of the inner ear and the air-filled space of the middle ear. This fistula is a passage for the flow of perilymph fluid into the middle ear compartment. PLF is broadly classified into two main categories of congenital or acquired. Main acquired etiologies include traumatic, erosive, iatrogenic and idiopathic [1]. Moreover, traumatic tears in the oval or round windows remain a major cause.

\section{Case Report}

A 46-year-old male presented to the ENT clinic following a mechanical fall in which the back of his head struck tiled flooring, resulting in a transient loss of consciousness. The following morning he woke up with profound left sided hearing loss, positional vertigo and unsteadiness. A bedside ENT examination revealed lateralization to the right ear on weber's test and a positive hallpike's manoeuvre with no focal neurological deficits. A pure tone audiogram showed moderate to severe sensorineural hearing loss on the left (Figure 1).

After two days the patient returned with worsening disequilibrium and rotatory vertigo. CT brain showed haemorrhagic contusions in the left temporal lobe. A CT scan of the petrous bone showed no evidence of fracture. The MRI of Internal Auditory Meatus (IAM) demonstrated normal cochlear and semicircular canals on both sides, with no evidence of effusion within the middle ear and clear mastoids.

Initially he was managed conservatively. His symptoms remained unchanged after two weeks. Therefore he underwent an exploratory tympanotomy. Intraoperatively suspected fistula in the region of the footplate and round window was patched using a temporalis fascia. Post-operatively his vertigo improved with no significant improvement in hearing.

\section{Discussion}

Traumatic PLF was first described by Fee [2]. In 1970 Stroud and Calcattera [3] first reported spontaneous PLF without trauma. Statistically trauma is the most common cause of PLF. It can be penetrating or blunt. Grimm et al. [1], in 1989 showed that even minor whiplash injuries alone can cause PLF. In fact one in three of his study population comprised of such cases. A few years later Fitzgerald reviewed 350 patients with PLF and showed that only $36 \%$ had direct head or ear trauma, which were often mild in nature without loss of consciousness [4]. Goodhill [5], presented three cases of oval and round window PLFs resulting from exertion. Therefore, an increase in pressure when applied to the correct place in the inner or middle ear can cause ruptures in the membranous labyrinth that can affect both hearing and balance [6].

The manifestations of this condition vary in severity and complexity, ranging from mild to incapacitating. PLF symptoms include fluctuating or stable hearing loss, tinnitus, aural fullness, vertigo, instability of gait, or a combination of these symptoms.

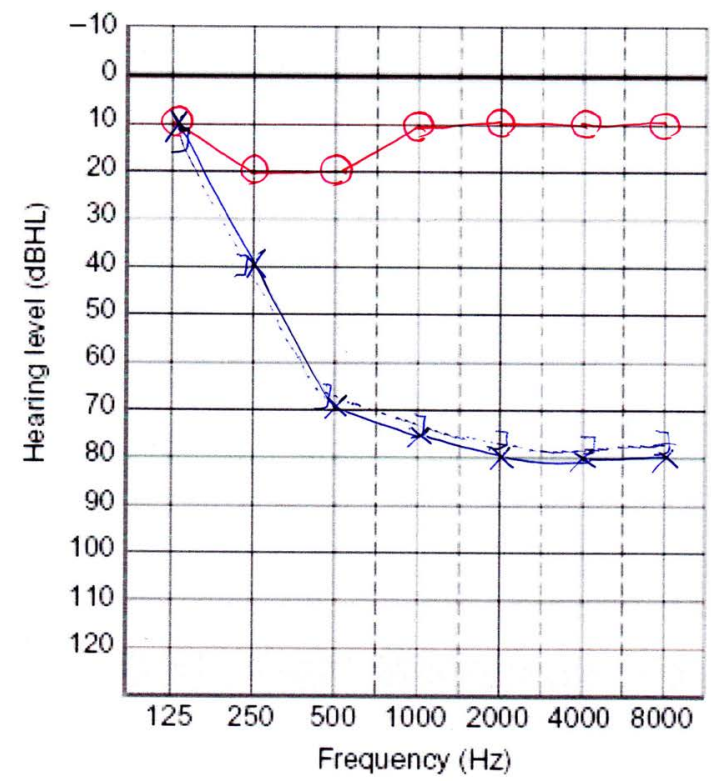

Figure 1: A pure tone audiogram showed moderate to severe sensorineural hearing loss on the left.

*Corresponding author: Ardalan Zolnourian, Department of ENT, Queen's Hospital Burton, Belvedere Rd, Burton-on-Trent, Staffordshire, DE13 ORB, UK, E-mail: z.ardalan@googlemail.com

Received September 03, 2013; Accepted September 25, 2013; Published September 27, 2013

Citation: Zolnourian A, Paramanathan V (2013) Perilymphatic Fistula Following a Closed Head Injury. J Trauma Treat 2: 177. doi:10.4172/2167-1222.1000177

Copyright: @ 2013 Zolnourian A, et al. This is an open-access article distributed under the terms of the Creative Commons Attribution License, which permits unrestricted use, distribution, and reproduction in any medium, provided the original author and source are credited. 
Unfortunately, many otolaryngologic and neurologic conditions can present with similar symptoms, particularly labyrinthine concussion and Meniere's disease [7].

Diagnosis of PLF remains challenging. Historically, labyrinthine rupture and its associated fistula were only recognised at a tympanotomy operation. A PLF was confirmed by inspecting the round and oval window for gross leakage of clear fluid; however, it is not always easy to make a definite diagnosis. An actual hole in a membrane is very rarely seen [8]. Its presence has to be inferred from a leak of fluid, and that leak may be very slow. Moreover, tissue fluid exudation or moistening of local anaesthetic injected into the meatus can easily be misinterpreted as perilymph [9].

Alternatively, sampling the suspected perilymph fluid for $\beta 2$ Transferrin can confirm the diagnosis. $\beta 2$-transferrin is a protein unique to cerebrospinal fluid, aqueous humor, and human perilymph. Bassiouny et al. [10] developed the advanced use of clinical electrophoreticassay of middle ear fluid for thepresence of $\beta 2$-transferrin. Weber et al. [11] reported the presence of $\beta 2$ - transferrin in six of nine operated ears in children with suspected perilymph fistulas. All 10 control patients tested negative for $\beta 2$-transferrin [11]. However this technique has diagnostic limitations. In particular dilutional effect of sample handling in preparation may lower $\beta 2$-transferrin concentration below the detectable limits of the assay [12] and low sensitivity of this technique [13]. Alternative diagnostic biomarker has also been suggested such as apolipoprotein-d, the use of which is doubtful [14].

Other useful diagnostic modalities include CT or MRI scans. CT scans can be used for evaluation of the bony otic capsule, temporal bone and ossicular chain integrity. It can also demonstrate other pathology such as contusions as in our case. Temporal bone fractures, mondini dysplasia, or dilated cochlear or vestibular aqueducts, which may indicate increased risk for perilymph fistula are other valuable diagnostic features. The MRI is mainly used to exclude alternative diagnoses to perilymph fistula. Morris et al. [15] used adult cats and created an experimental cochlear fistula. They then demonstrated the usefulness of pre and post-contrast MRI scans following injection of intrathecal gadolinium infusion to confirm and localize the presence of PLFs. Post-contrast images of the fistualized cochlea demonstrated a significant increase in the signal intensity of the cochlear perilymph with pooling of enhanced perilymph observed in the ipsilateral mastoid bulla. Although effective, this is both expensive and invasive [15].

Other diagnostic methods include Jugular venous compression [8], fluorescein injection into cerebrospinal fluid [16] and Electrocochleography [17]. Nevertheless, most of the mentioned diagnostic tests lack in both sensitivity and specificity. Although subjective, direct inspection and visualization of the oval and round windows intraoperatively remains a reliable method of diagnosis. Perilymph can often be seen around the windows or in the middle ear cavity.

Management of PFLs remains a controversial issue. However, most clinicians seem to agree on an initial period of conservative management involving bed rest, head elevation, stool softeners, avoidance of valsalva's maneuver [18] and close observation for the first 24-72 hours. This allows sufficient time for all membrane leaks (labyrinthine concussion) to heal [7].

Opinion around timing of surgery is divided. Some believe that exploratory surgery should be reserved for patients with cerebrospinal fluid leakage, progressive sensorineural hearing loss, vestibular symptomatology [19], intractable vertigo, radiologic evidence of PLF and meningitis. [20]. Spontaneous healing of fistulas is related to the severity of trauma; the more severe the trauma the less chance of healing [21]. Finally moving forward to surgical exploration and grafting is a decision that should be determined by the clinical history and symptoms. In this case study surgical approach was chosen in the context of ongoing vestibular symptoms and sensorineural hearing loss despite the initial conservative management.

Even after surgery symptoms may not disappear altogether. Hearing is less likely to improve or be restored compared with balance disturbances after surgical repair. Seltzer and McCabe reported that $49 \%$ of treated ears improved in terms of auditory function, but only $23 \%$ improved to serviceable hearing levels, and $11 \%$ had continued hearing deterioration. [22]. Friedland and Wackym [23] reviewed the surgical cases at several institutions and showed overall improvement rate in vestibular symptomatology approximating $90 \%$, which was also noted in this case, whereas stabilization of hearing was only achieved in almost $50 \%$ of patients, and improvement of hearing in a much lower percentage.

The fistula that follows damage to one of the window membranes is not the sole, or indeed the main cause of the inner ear disturbance, since the basilar membrane, Reissner's membrane and other intralabyrinthine structures may be damaged [9]. This explains the poor hearing this patient continued to suffer despite effective sealing of leaking perilymph.

\section{Conclusion}

Post-traumatic PLF is a known clinical entity. The most common presenting features include hearing loss and vertigo. Diagnosis is challenging, however, intraoperative assessment remains a gold standard. Diagnosis can be aided using CSF biomarkers, high resolution CT and MRI. The initial management is often conservative, however, in the setting of trauma exploratory surgery with oval and round window patching should be considered at early stage. Surgical treatment remains an effective method of treatment, but its effect on hearing loss is less predictable.

\section{References}

1. Grimm RJ, Hemenway WG, Lebray PR, Black FO (1989) The perilymph fistula syndrome defined in mild head trauma. Acta Otolaryngol Suppl 464: 1-40.

2. Fee GA (1968) Traumatic perilymphatic fistulas. Arch Otolaryngol 88: 477-480

3. Stroud MH, Calcaterra TC (1970) Spontaneous perilymph fistulas. Laryngoscope 80: 479-487.

4. Fitzgerald DC (1996) Head trauma: hearing loss and dizziness. J Trauma 40: 488-496.

5. Goodhill V (1971) Sudden deafness and round window rupture. Laryngoscope 81: $1462-1474$.

6. Wall C 3rd, Rauch SD (1995) Perilymph fistula pathophysiology. Otolaryngol Head Neck Surg 112: 145-153.

7. Kim SH, Kazahaya K, Handler SD (2001) Traumatic perilymphatic fistulas in children: etiology, diagnosis and management. Int J Pediatr Otorhinolaryngol 60: $147-153$

8. Vartiainen E, Nuutinen J, Karjalainen S, Nykänen K (1991) Perilymph fistula--a diagnostic dilemma. J Laryngol Otol 105: 270-273.

9. Mawson S, Ludman H (1979) Diseases of the ear a textbook of otology 4 470-471.

10. Bassiouny M, Hirsch BE, Kelly RH, Kamerer DB, Cass SP (1992) Beta 2 transferrin application in otology. Am J Otol 13: 552-555.

11. Weber PC, Kelly RH, Bluestone CD, Bassiouny M (1994) Beta 2-transferrin 
Citation: Zolnourian A, Paramanathan V (2013) Perilymphatic Fistula Following a Closed Head Injury. J Trauma Treat 2: 177. doi:10.4172/21671222.1000177

Page 3 of 3

confirms perilymphatic fistula in children. Otolaryngol Head Neck Surg 110 381-386

12. Rauch SD (2000) Transferrin microheterogeneity in human perilymph Laryngoscope 110: 545-552.

13. Levenson MJ, Desloge RB, Parisier SC (1996) Beta-2 transferrin: limitations of use as a clinical marker for perilymph. Laryngoscope 106: 159-161.

14. Teilan SA, Disher MJ, Sun Q (1998) Biochemical markers for identification of human perilymph. Abstr Am Otol Soc 131: 39.

15. Morris MS, Kil J, Carvlin MJ (1993) Magnetic resonance imaging of perilymphatic fistula. Laryngoscope 103: 729-733.

16. Arenberg IK, Mischke RE, Gibson WP (1993) Negative observation of intravenously administered fluorescein in perilymph at stapes surgery. Am J Otol 14: 95-96.

17. Meyerhoff WL, Yellin MW (1990) Summating potential/action potential ratio in perilymph fistula. Otolaryngol Head Neck Surg 102: 678-682.
18. Yanagihara N, Nishioka I (1987) Pneumolabyrinth in perilymphatic fistula: report of three cases. Am J Otol 8: 313-318.

19. Prisman E, Ramsden JD, Blaser S, Papsin B (2011) Traumatic perilymphatic fistula with pneumolabyrinth: diagnosis and management. Laryngoscope 121 856-859.

20. Maitland CG (2001) Perilymphatic fistula. Curr Neurol Neurosci Rep 1: 486491.

21. Singleton GT (1986) Diagnosis and treatment of perilymph fistulas without hearing loss. Otolaryngol Head Neck Surg 94: 426-429.

22. Seltzer S, McCabe BF (1986) Perilymph fistula: the lowa experience. Laryngoscope 96: 37-49.

23. Friedland DR, Wackym PA (1999) A critical appraisal of spontaneous perilymphatic fistulas of the inner ear. Am J Otol 20: 261-276. 